\title{
The Manufacturing of a Multi-surface Monolithic Telescope with Freeform Surfaces
}

\author{
Todd Blalock, Brian Myer, Brittany Cox, Jessica Nelson \\ Optimax Systems Inc., 6367 Dean Parkway, Ontario, New York 14519, USA
}

\begin{abstract}
Monolithic multi-surface telescopes combined with freeform optical surfaces provide improvements in optical performance in a smaller footprint as compared to systems with spherical surfaces, while providing superior mechanical stability to traditional telescope assemblies. Three different monolithic telescope concepts, in different configurations and optical performance were produced as proof of concepts. The results of the telescopes are presented as well as the unique manufacturing and testing challenges to produce these monolithic optical systems.
\end{abstract}

\section{Advantages for monolithic optical systems}

Monolithic systems offer the advantage of high stability as compared to traditional multi-element design. Alignment stability is crucial in high G-force applications such as airborne and spaceborne systems. The risk of element misalignment is greatly reduced by eliminating the need for opto-mechanical fixturing required to mount and position the optics.

\section{Optical and mechanical design}

In telescope systems off-axis freeform configurations are desirable when high contrast (no central obstruction) and reduced footprint is desired.[1,2] Recent improvements in optical freeform manufacturing have allowed for increased optical performance in off-axis optical systems which is particularly applicable in these monolithic structures.[3]

The designs of the three monolithic telescopes were a collaboration between Optimax Systems and NASA's Goddard Space Flight Center. Figure 1 shows the embodiment of all three monoliths. Figure 1 (a) is a twofreeform mirror solid glass prism, Figure 1 (b) is a threefreeform, diffraction limited, solid glass monolith, and Figure 1 (c) is a lightweight, two freeform mirror system. Both solid glass telescopes consist of a single blank of high purity fused while the light-weighted version uses a stable mirror grade glass substrate. All mirrored surfaces were given freeform prescriptions governed by $\mathrm{XY}$ polynomials. All three telescopes were designed with a field of view of $\pm 4.3^{\circ}$ in $\mathrm{Y}$-axis and $\pm 1.4^{\circ}$ in $\mathrm{X}$-axis at $\mathrm{f} / 3.4$. The two-freeform solid glass telescope shown in Figure 1(a) has four faces, faces 1 and 4 are respective plano entrance and exit faces and surfaces 2 and 3 are the freeform surfaces that provide the optical power. The entire telescope assembly is compact enough to fit within a single unit (1U) of a CubeSat payload with a weight of $1.3 \mathrm{~kg}$. This design was not optimized to provide diffraction limited performance.

The next design iteration was the three freeform high-resolution solid glass telescope shown in Figure 1(b) which added a third freeform surface to provide diffraction limited performance. The additional glass material required to produce the increased optical performance contributes to its significant mass of $\sim 2.3 \mathrm{~kg}$.

The final design form in this study is the lightweight version shown in Figure 1(c). This light-weighted telescope contains the same optical prescription as the initial off-axis monolith design, however the light rays no longer propagate through any optical glass. This design reduced the weight by $60 \%$ to $0.42 \mathrm{~kg}$.

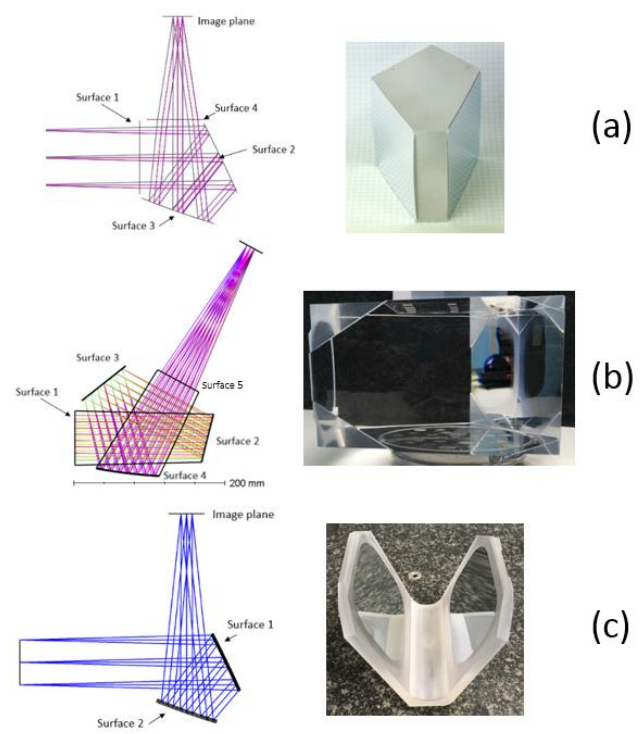

Figure 1. The three embodiments of monolithic telescopes shown with optical ray trace (left) and photograph of finished part (right). (a) Two freeform surface, solid prism, (b) diffraction limited, solid prism, and (c) two freeform, lightweight system.

* Corresponding author: tblalock@optimaxsi.com 


\section{Manufacturing and testing}

\subsection{Manufacturing considerations}

All three monolithic telescope designs were polished on Optimax's custom and proprietary robotic freeform polishing platform $[4,5]$. During the manufacturing process it is imperative that a consistent global coordinate system be defined to reference the freeform surfaces onto the monolithic structure. This coordinate system is usually based on easily accessible features built into the monolith. These features, or fiducials, are referenced during the manufacturing and metrology steps to ensure proper form of the freeform surface as well as its global position in 3D space. Figure 2 shows an example coordinate system for the lightweight monolith.

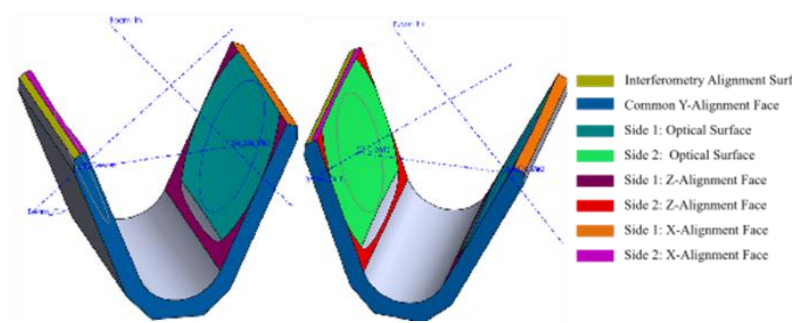

Figure 2: Fiducial system for each side of the light weight monolith.

In the case of the lightweight monolith, additional manufacturing challenges included the design of miniaturized tooling. Due to the structure and shape of the lightweight monolith, there are issues with accessibility of the polishing tool to reach tight regions near the dihedral of the two surfaces. Due to the limited work space special care was taken to verify robot tool path to avoid collision between tooling and the part.

\subsection{Testing considerations}

Freeform metrology primarily relies on a two-phase approach using both contact metrology methods to locate the freeform surface within a reference system as well as a higher resolution measurement of the optical freeform surface. We mention several freeform metrology techniques used during the manufacturing and testing of the monolithic freeform telescopes that include two contact methods: coordinate measurement machine and a high resolution profilometer (Figure 3), as well as a noncontact method: deflectometry (Figure 4). [6]

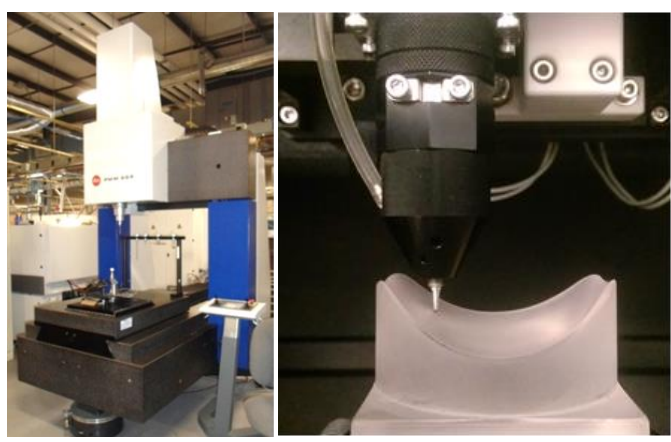

Figure 3. CMM and UA3P at Optimax.

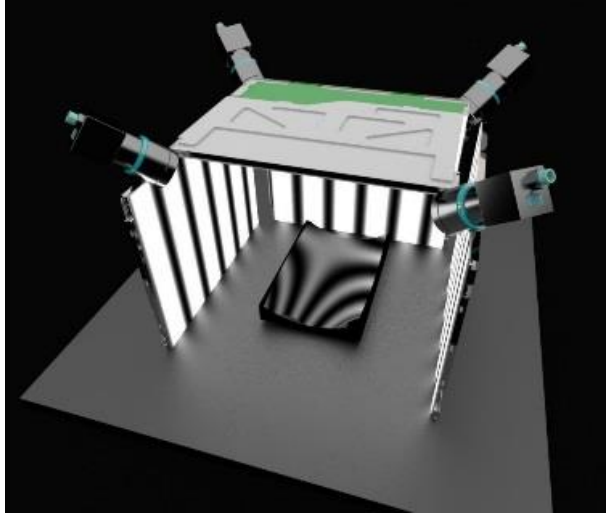

Figure 4. Rendering of deflectometry system at Optimax.

Form and surface position is adjusted using CMM feedback down to a few micrometers of residual error. Then the surface can be evaluated, best fit, using deflectometry and UA3P. These can reveal MSF errors due to sub-aperture raster polishing, which must be removed via smoothing.
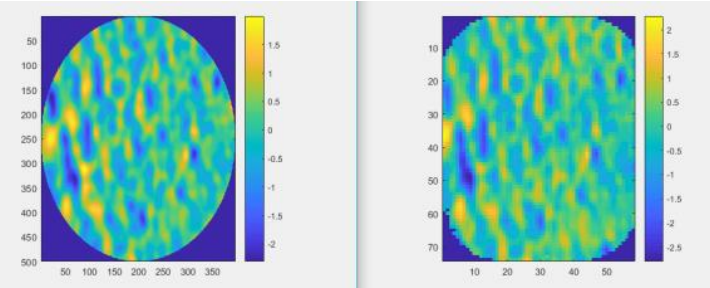

Figure 5. In-process deflectometry and UA3P data for lightweight monolith freeform surface.

\section{Conclusions :}

Innovation in optical design, datum control, manufacturing and metrology are required to create high quality freeform monolithic telescopes for lightweight, flight applications.

\section{References:}

1. K.Fuerschbach et al., "Assembly of a freeform offaxis optical system employing three p-polynomial Zernike mirrors," Optics Letters 39, 2896-2899 (2014).

2. J. M. Howard et al., "Improving the performance of three-mirror imaging systems with Freeform Optics," in Freeform Optics 2013, OSA Technical Digest, (The Optical Society, 2013), paper FT2B.6

3. J. L. Lawson et al., "Freeform monolithic multisurface telescope manufacturing" in Optical Design Fabrication 2017 (The Optical Society, 2017), paper JTH1C.2

4. T. Lynch et al., "Deterministic form correction of extreme freeform optical surfaces" Proc. SPIE 9633 , Optifab 2015, 96331F

5. D. Brooks et al., "Manufacturing of a large, extreme freeform, conformal window with robotic polishing," Proc. SPIE 10742, Optical Manufacturing and Testing XIII, 107420L

6. T. Blalock et al., "Metrology of Freeform Optics," Optical Design and Fabrication 2017 (The Optical Society, 2017), paper JTh4B.1 